\title{
The nonlinear thermomechanical vibration of a functionally graded beam on Winkler-Pasternak foundation
}

\author{
Vasile Marinca $^{1,2}$, and Nicolae Herisanu ${ }^{1,2, *}$ \\ ${ }^{1}$ University Politehnica Timisoara, 300222 Bd. Mihai Viteazu 1, Timisoara, Romania \\ ${ }^{2}$ Center for Advanced Technical Research, Romanian Academy Branch of Timisoara, Bd. Mihai Viteazu 24, Timisoara, Romania
}

\begin{abstract}
By using the Optimal Auxiliary Functions Method (OAFM), nonlinear free thermomechanical vibration of functionally graded beam (FGB) on Winkler-Pasternak elastic foundation is studied. Based on von Karman geometric nonlinearity, on Euler-Bernoulli beam theory and also on Galerkin procedure we obtain a second-order nonlinear differential equation with quadratic and cubic nonlinear terms. The results obtained by means of OAFM are compared and shown to be in an excellent agreement with available solutions known in the literature.
\end{abstract}

\section{Introduction}

The concept of functionally graded materials (FGM) appeared in 1984 in Sendai area of Japan. These are heterogeneous, anisotropic materials and are made from a mixture of ceramics and metals. The mechanical properties of them varies smoothly and continuously from the surface to other surface of the material. Initially FGM were designed as thermal barrier materials for aerospace application and fusion reactors. These materials had found application in various fields of engineering like automotive, semiconductor industry, manufacturing industry, biomedical science, aerospace, defense industry and general structural element in thermal environments.

Many researchers have investigated different aspects of FGM. The effect of damage on free and forced vibration of a FG cantilever beam is studied by Birman and Byrd [1]. The modes of damage include a region with degraded stiffness adjacent to the root of the beam, a single delamination crack and a single crack at the root cross section of the beam propagating in the thickness direction. Dokmeci presented a system of 1-D equations so that to analyze the thermoviscoelastic behavior of an axially FGB of rectangular cross section at highfrequency vibration [2]. The system of 1-D equation governs the extensional, flexural, torsional and thickness shear and also the coupled vibrations of the beam at high frequency. Khorrambadi [3] analyzed the free vibration of FGB with piezoelectric actuators subjected to axial compressive loads. The elasticity modulus of beam is assumed to vary as a power form of the thickness coordinate variable. The effect of the applied voltages, axial compressive loads and FG index of the vibration frequency are discussed. The static Green's functions for
FG Euler-Bernoulli and Timoshenko beams are presented by Carl et al [4]. All material properties are arbitrary functions along the beam thickness direction. For symmetrical material properties along the beam thickness directions and symmetric cross-sections, the resulting stress distribution is also symmetric. Alshabatat and Naghshineh presented in [5] a design method to optimize the material distribution of FGM with respect to some vibration and acoustic properties. Two novel volume fraction laws are used to describe the material volume distributions through the length of the FGB. Ke et al [6] discussed the effects of material property distribution and end supports on the nonlinear dynamic behavior of FGB. The direct numerical integration method and Runge-Kutta method are employed to find the nonlinear vibration of FGB with different end supports. Yaghobi and Torabi [7] investigated an analytic solution using variational iteration method for nonlinear vibration and post-buckling of beams made of FGM resting on a nonlinear elastic foundation, subject to an axial force. Mohammadi [8] considered the non-linear terms in von-Karaman's strain-displacement relation with the help of Hamilton's principle. The equation of potential and kinetic energy of the beam are derived and as a result, the nonlinear motion equation could be reached. Fu et al [9] used the finite difference method or dynamic equations of FGB with piezoelectric patches. The Eshelby-Mori-Tanaka approach based on an equivalent fiber is used by Thomas et al [10] to investigate free vibration of functionally graded nanocomposite beam reinforced by randomly oriented straight single-walled carbon nanotubes. The first five normalized mode shapes for this type of beam with different boundary conditions and different carbon nanotubes orientation are presented. The large-amplitude free vibration of clamped immovable thin beams made of FGM is investigated by Elmaguiri et al [11] using the

\footnotetext{
* Corresponding author: nicolae.herisanu@upt.ro
} 
energy method and a multimode approach. By means of harmonic balance method, the equations of motion are converted into a nonlinear algebraic form and are solved by an iterative numerical method.

The linear and nonlinear vibration behavior of monomorph and bimorph beam made from a mixture of PZT4 and PZT-5H with material composition are investigated by Yang et al [12]. Maganti et Nalluri [13] considered the deformation variables to determine flapwise bending of rotating functionally graded double tapered beam attached to a rigid hub. The equations of motions are derived using hybrid deformation variables employing Lagrange's approach and Rayliegh-Ritz method is used to evaluate the frequencies of the beam. The effect of temperature field on the natural frequencies of FGB with different conditions is studied by Kashyzadeh and Asforjani [14]. Modal analysis has been performed for a FGB with clamped-clamped and clamped-free supports. The vibration of Euler-Bernoulli beam with FGM which is modeled by fourth-order partial differential equations with variable coefficients are examined by Yigit et al [15] by using the Adomian Decomposition Method. Su et al [16] presented a unified solution for free and transient analyses of a functionally graded piezoelectric curved beam with general boundary conditions within the framework of Timoshenko beam theory. The formulation is derived by means of the variational principle in conjunction with a modified Fourier series. Fundamental frequency of sandwich beams with functionally graded face sheet and homogenous core is studied by Mhu and Zhao [17].

The classical plate theory is used to analyze the face sheet and a higher-order theory is used to analyze the core of sandwich beams in which both the transverse normal and shear strains of the core are considered. Shwartsman and Majak [18] studied free vibration of axially functionally graded Euler-Bernoulli beams with elastically restrained ends. The method of initial parameters in differential form is treated for the numerical solution of the problem. Numerical method proposed has fourth order of accuracy and the Richardson extrapolation of results with different step sizes gives solutions of the sixth order of accuracy.

In the present paper are investigated the nonlinear free thermomechanical vibrations of FGB on WinklerPasternak elastic foundation. Considering von Karaman geometric nonlinearity, the Euler-Bernoulli beam theory and Galerkin procedure, we obtain a second-order nonlinear differential equation with quadratic and cubic nonlinear terms. This equation is solved by using a new analytical approximate approach - OAFM. The accuracy of the analytical results obtained through the proposed approach is proved by numerical simulations developed in order to validate analytical results. Several numerical examples show that the proposed procedure is simple and easy to use.

\section{The governing equations}

In what follows, we consider a FGB of length $L$, width $b$, and thickness $h$ resting on an elastic foundation of Winkler-Pasternak type and subjected to an axial force $P$ (fig.1).

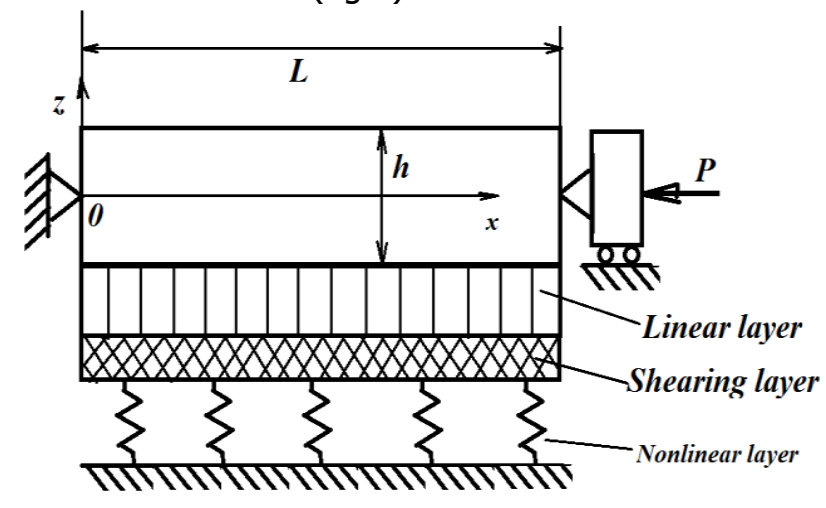

Fig.1. Schematic of the FGB with nonlinear foundation

The mechanical properties of the FGB can be varied as a function along thickness, based on the rule of mixtures. Taking into account the rule of mixtures, we have

$$
\begin{aligned}
& E(z)=\left(E_{2}-E_{1}\right)\left(\frac{2 z+h}{2 h}\right)^{k}+E_{1} \\
& \rho(z)=\left(\rho_{2}-\rho_{1}\right)\left(\frac{2 z+h}{2 h}\right)^{k}+\rho_{1} \\
& v(z)=\left(v_{2}-v_{1}\right)\left(\frac{2 z+h}{2 h}\right)^{k}+v_{1}
\end{aligned}
$$

where subscript 1 and 2 denote the top surface $(z=-h / 2)$ and bottom surface $(\mathrm{z}=\mathrm{h} / 2)$ respectively. The constant $\mathrm{k}$ characterizes the distributions of material properties. The case $\mathrm{k}=0$ corresponds to an isotropic homogenous beam.

For a small strain, moderate deformation and rotation, the axial strain of the midplane of the beam accounting for the midplane stretching is given by [19]:

$$
\varepsilon_{x}=\frac{\partial \bar{U}}{\partial \bar{x}}+z \frac{\partial^{2} \bar{W}}{\partial \bar{x}^{2}}+\frac{1}{2}\left(\frac{\partial \bar{W}}{\partial \bar{x}}\right)^{2}
$$

where, based on Euler-Bernoulli beam theory, the displacement of an arbitrary point along the $\mathrm{x}$ and $\mathrm{z}$ axes are $\bar{U}(x, z, t)$ and $\bar{W}(x, z, t)$ respectively. If $U(x, t)$ and $W(x, t)$ are displacement components in the midplane, then we have

$$
\begin{gathered}
\bar{U}(x, z, t)=U(x, t)+z \frac{\partial W}{\partial x} \\
\bar{W}(x, z, t)=W(x, t)
\end{gathered}
$$

where $\mathrm{t}$ is the time. The normal stress for the von Karaman type of geometry is given by the law

$$
\sigma_{x x}=\frac{E(z)}{1-v^{2}(z)}\left[\frac{\partial \bar{U}}{\partial \bar{x}}+z \frac{\partial^{2} \bar{W}}{\partial \bar{x}^{2}}+\frac{1}{2}\left(\frac{\partial \bar{W}}{\partial \bar{x}}\right)^{2}\right]
$$


The curvature of the beam is given by

$$
\frac{1}{R}=\frac{\partial^{2} \bar{W}}{\partial \bar{x}^{2}}
$$

The axial, coupling and bending stiffness are defined by

$$
\begin{aligned}
A_{11} & =\int_{-h / 2}^{h / 2} \frac{E(z)}{1-v^{2}(z)} d z \\
B_{11} & =\int_{-h / 2}^{h / 2} \frac{E(z)}{1-v^{2}(z)} z d z \\
D_{11} & =\int_{-h / 2}^{h / 2} \frac{E(z)}{1-v^{2}(z)} z^{2} d z
\end{aligned}
$$

Assuming that the initial temperature of the beam is zero, resultant force and thermal momentum are defined as

$$
\begin{aligned}
& N_{T x}=-\int_{-h / 2}^{h / 2} \frac{E(z)}{1-v^{2}(z)} \alpha(z) T d z \\
& M_{T x}=-\int_{-h / 2}^{h / 2} \frac{E(z)}{1-v^{2}(z)} \alpha(z) T z d z
\end{aligned}
$$

The total induced axial force $\mathrm{N}_{\mathrm{x}}$ and bending moment $\mathrm{M}_{\mathrm{x}}$ are related to the stress resultants as

$$
\begin{aligned}
& N_{x}=A_{11}\left[\frac{\partial \bar{U}}{\partial \bar{x}}+\frac{1}{2}\left(\frac{\partial \bar{W}}{\partial \bar{x}}\right)^{2}\right]+B_{11} \frac{\partial^{2} \bar{W}}{\partial \bar{x}^{2}}+N_{T \bar{x}} \\
& M_{x}=B_{11}\left[\frac{\partial \bar{U}}{\partial \bar{x}}+\frac{1}{2}\left(\frac{\partial \bar{W}}{\partial \bar{x}}\right)^{2}\right]+D_{11} \frac{\partial^{2} \bar{W}}{\partial \bar{x}^{2}}+M_{T \bar{x}}
\end{aligned}
$$

The equations of motion for axial and transverse vibration of FGB based on Euler-Bernoulli beam theory and von Karaman geometric nonlinearity are

$$
\begin{gathered}
I_{1} \frac{\partial \bar{U}}{\partial t}-\frac{\partial N_{x}}{\partial \bar{x}}=0 \\
\frac{\partial^{2} M_{x}}{\partial \bar{x}^{2}}-\frac{\partial}{\partial \bar{x}}\left(N_{x} \frac{\partial \bar{W}}{\partial \bar{x}}\right)+I_{1} \frac{\partial^{2} \bar{W}}{\partial t^{2}}=F_{w}
\end{gathered}
$$

where

$$
I_{1}=\int_{-h / 2}^{h / 2} \rho(z) d z
$$

and $F_{w}$ is reaction of the elastic Winkler-Pasternak foundation.

Now, if the axial inertia is neglected, then from Eq.(14) gives
$N_{x}=N_{x 0}=A_{11}\left[\frac{\partial \bar{U}}{\partial \bar{x}}+\frac{1}{2}\left(\frac{\partial \bar{W}}{\partial \bar{x}}\right)^{2}\right]+B_{11} \frac{\partial^{2} \bar{W}}{\partial \bar{x}^{2}}+N_{T \bar{x}}$

and therefore $\mathrm{N}_{\mathrm{x}}$ is independent of $\bar{x}$. From Eq.(17) we obtain

$$
A_{11}^{-1}\left(N_{x}-B_{11} \frac{\partial^{2} \bar{W}}{\partial \bar{x}^{2}}-N_{T \bar{x}}\right)-\frac{1}{2}\left(\frac{\partial \bar{W}}{\partial \bar{x}}\right)^{2}=\frac{\partial \bar{U}}{\partial \bar{x}}
$$

Supposing that the beam has immovable ends,

$$
\bar{U}(0, t)=\bar{U}(L, t)=0
$$

and integrating Eq.(20) with respect to $\bar{x}$ and having in attention that $\mathrm{N}_{\mathrm{x}}$ is independent of $\bar{x}$, we obtain

$$
\begin{aligned}
& N_{x}=\frac{A_{11}}{2 L} \int_{0}^{L} \bar{W}^{\prime 2} d x+ \\
& +\frac{B_{11}}{L}\left[\bar{W}^{\prime}(L, t)-\bar{W}^{\prime}(0, t)\right]+N_{T \bar{x}}-P
\end{aligned}
$$

From Eqs.(14) and (22) results

$$
\frac{\partial \bar{U}}{\partial \bar{x}}=A_{11}^{-1}\left(N_{x}-N_{T x}\right)-\frac{1}{2} \bar{W}^{\prime 2}-B_{11} \bar{W}^{\prime \prime}
$$

or

$$
\begin{aligned}
& \frac{\partial \bar{U}}{\partial \bar{x}}=\frac{1}{2 L} \int_{0}^{L} \bar{W}^{\prime 2} d x+\frac{B_{11}}{A_{11} L}\left[\bar{W}^{\prime}(L, t)-\bar{W}^{\prime}(0, t)\right]- \\
& -\frac{1}{2} \bar{W}^{\prime 2}-\frac{B_{11}}{A_{11}} \bar{W}^{\prime \prime}
\end{aligned}
$$

Differentiating Eq.(24) with respect to $\bar{x}$ yields

$$
\frac{\partial^{2} \bar{U}}{\partial \bar{x}^{2}}=-\bar{W}^{\prime} \bar{W}^{\prime \prime}-\frac{B_{11}}{A_{11}} \bar{W}^{\prime \prime \prime}
$$

where prime denotes derivative with respect to $\bar{x}$.

Substituting Eq.(24) into Eq.(15), we obtain

$$
\begin{aligned}
& M_{x}=\frac{B_{11}}{2 L} \int_{0}^{L} \bar{W}^{\prime 2} d x+\frac{B_{11}^{2}}{A_{11} L}\left[\bar{W}^{\prime}(L, t)-\bar{W}^{\prime}(0, t)\right]- \\
& -\frac{B_{11}}{A_{11}} \bar{W}^{\prime \prime}+D_{11} \bar{W}
\end{aligned}
$$

Furthermore, using Eqs.(15) and (26) we have

$$
\frac{\partial^{2} M}{\partial \bar{x}^{2}}=\left(D_{11}-\frac{B_{11}^{2}}{A_{11} L}\right) \bar{W}^{(I V)}
$$

By replacing the Eqs.(22) and (27) into Eq.(17), the governing nonlinear thermomechanical vibration equation of FGM is obtained as follows 


$$
\begin{aligned}
& I \bar{W}^{\prime \prime}+\left(D_{11}-\frac{B_{11}^{2}}{A_{11} L}\right) \bar{W}^{(I V)}-\bar{W}^{\prime \prime}\left[\frac{A_{11}}{2 L} \int_{0}^{L} \bar{W}^{\prime 2} d x+\right. \\
& \left.+\frac{B_{11}}{L}\left(\bar{W}^{\prime}(L, t)-\bar{W}^{\prime}(0, t)\right)+N_{T x}-P\right]=F_{W}
\end{aligned}
$$

where the dot denotes derivative with respect to time.

Due to the nonlinear elastic Winkler-Pasternak foundation, the second side of the Eq.(8) is defined as follows:

$$
F_{W}=-k_{L} \bar{W}-k_{N L} \bar{W}^{3}+k_{s} \frac{\partial^{2} \bar{W}}{\partial \bar{x}^{2}}
$$

where $\mathrm{k}_{\mathrm{L}}$ and $\mathrm{k}_{\mathrm{NL}}$ are the linear and nonlinear coefficients respectively and $\mathrm{k}_{\mathrm{s}}$ is the shear coefficient of elastic foundation. In order to derive general results independent of dimensions and specific sizes, we introduce the dimensionless parameters:

$$
\begin{gathered}
x=\frac{\bar{x}}{L}, \quad W=\frac{\bar{W}}{h}, \quad r=\sqrt{\frac{I_{1}}{A}}, \quad 2 \alpha=\frac{A_{11} r^{2}}{D_{11}-\frac{B_{11}^{2}}{A_{11}}} \\
t=\frac{\bar{t}}{L} \sqrt{\frac{1}{D_{11}}\left(D_{11}-\frac{B_{11}^{2}}{A_{11} L}\right)}, N_{T}=\frac{N_{T \bar{x}} L^{2}}{D_{11}-\frac{B_{11}^{2}}{A_{11}}}, \\
P=\frac{\bar{P} L^{2}}{D_{11}-\frac{B_{11}^{2}}{A_{11}}, \quad \lambda=\frac{B_{11} r^{2}}{D_{11}-\frac{B_{11}^{2}}{A_{11}}}, k_{1}=\frac{k_{L} L^{4}}{D_{11}-\frac{B_{11}^{2}}{A_{11}}}} \\
k_{2}=\frac{k_{N L} r^{2} L^{4}}{D_{11}-\frac{B_{11}^{2}}{A_{11}}}, \quad k_{3}=\frac{k_{s} L^{2}}{D_{11}-\frac{B_{11}^{2}}{A_{11}}}+\alpha\left(P-N_{T}\right)
\end{gathered}
$$

By replacing Eq.(30) into Eq.(28) it results

$$
\begin{aligned}
& W^{\prime \prime}+W^{(I V)}+k_{1} W+k_{2} W^{3}-k_{3} W^{\prime \prime}- \\
& -\alpha W^{\prime \prime}\left[\int_{0}^{1} W^{\prime 2} d x+\lambda\left(W^{\prime}(1, t)-W^{\prime}(0, t)\right)\right]=0
\end{aligned}
$$

The Eq.(31) is a partial differential equation in two dimension, displacement $\mathrm{x}$ and time $\mathrm{t}$. Using the Galerkin method, Eq.(31) becomes an ordinary differential equation. Assuming that the transverse displacement is expressed as

$$
W(x, t)=X(x) T(t)
$$

where $\mathrm{X}(\mathrm{x})$ is the linear fundamental vibration mode and $\mathrm{T}(\mathrm{t})$ is the time dependent function to be determined. Substituting Eq.(32) into Eq.(31) and applying Galerkin's method, in which the orthogonality property of the mode shapes is used, yields:

$$
\ddot{T}+a T+b T^{2}+c T^{3}=0
$$

where

$$
\begin{aligned}
& a=\int_{0}^{1} X(x) X^{(I V)}(x) d x+k_{1} \int_{0}^{1} X^{2}(x) d x-k_{3} \int_{0}^{1} X(x) X^{\prime \prime}(x) d x \\
& b=-\alpha \lambda\left[X^{\prime}(1)-X^{\prime}(0)\right] \int_{0}^{1} X(x) X^{\prime \prime}(x) d x \\
& c=k_{2} \int_{0}^{1} X^{(I V)}(x) d x-\alpha\left(\int_{0}^{1} X^{\prime 2}(x) d x\right)\left(\int_{0}^{1} X(x) X^{\prime \prime}(x) d x\right)
\end{aligned}
$$

For the case of simply supported beam, the fundamental vibration mode is

$$
X(x)=\sqrt{2} \sin \pi x
$$

The Eq.(33) contains a quadratic nonlinear term due to the presence of bending-extension coupling effect in FGB and cubic nonlinear term due to the WinklerPasternak foundation. This equation does not have an exact solution, but by means of OAFM may be obtained an approximate solution using a set of auxiliary functions. We remark that in Eq. (33) there exists no small or large parameter.

The beam centroid is subjected to the following initial conditions

$$
T(0)=A, \quad \dot{T}(0)=0
$$

\section{The Optimal Auxiliary Functions Method}

In the following, we consider the general form of a nonlinear differential equation

$$
L[T(t)]+N[T(t)]=0, \quad t \in D
$$

with the initial/boundary conditions

$$
B\left(T(t), \frac{d T(t)}{d t}\right)=0
$$

where $\mathrm{L}$ is a linear operator and $\mathrm{N}$ is a nonlinear operator, $\mathrm{t}$ denotes the independent variable and $\mathrm{T}(\mathrm{t})$ is an unknown function.

It is known that an exact solution for strongly nonlinear differential equations of type (37) and (38) is frequently hard to be found. To find an approximate solution, we suppose that this solution can be expressed in the form

$$
\bar{T}\left(t, C_{i}\right)=T_{0}(t)+T_{1}\left(t, C_{i}\right), \quad i=1,2, \ldots, n
$$

where the initial and the first approximation will be determined as described in what follows.

Substituting Eq.(39) into (37), it results in

$$
L\left[T_{0}(t)\right]+L\left[T_{1}\left(t, C_{i}\right]+N\left[T_{0}(t)+T_{1}\left(t, C_{i}\right)\right]=0\right.
$$


The initial approximation $\mathrm{T}_{0}(\mathrm{t})$ can be identified from the linear equation

$$
L\left[T_{0}(t)\right]=0
$$

with the initial/boundary conditions

$$
B\left(T_{0}, \frac{d T_{0}}{d t}\right)=0
$$

and the first approximation from the equation

$$
\begin{gathered}
L\left[T_{1}\left(t, C_{i}\right)\right]+N\left[T_{0}(t)+T_{1}\left(t, C_{i}\right)\right]=0, \quad i=1,2, \ldots, n \\
B\left(T_{1}, \frac{d T_{1}}{d t}\right)=0
\end{gathered}
$$

The nonlinear term in the last equation is expanded in the form

$$
N\left[T_{0}(t)+T_{1}\left(t, C_{i}\right)\right]=N\left[T_{0}(t)\right]+\sum_{k=1}^{\infty} \frac{T_{1}^{k}}{k !} N^{(k)}\left[T_{0}(t)\right]
$$

To avoid the difficulties that appear in solving the nonlinear differential equation (43), and to accelerate the rapid convergence of the first approximation $T_{1}$, instead of the last term arising in (43), we propose another expression such that Eq.(43) can be written in a new form

$$
\begin{aligned}
& L\left[T_{1}\left(t, C_{i}\right)\right]+A_{1}\left(T_{0}(t), \dot{T}_{o}(t), C_{j}\right) P\left[N\left(T_{0}(t)\right)\right]+ \\
& +A_{2}\left(T_{0}, \dot{T}_{0}, C_{k}\right)=0 \\
& B\left(T_{1}\left(t, C_{i}\right), \frac{d T_{1}\left(t, C_{i}\right)}{d t}\right)=0
\end{aligned}
$$

where $A_{1}$ and $A_{2}$ are two arbitrary auxiliary functions depending on the initial approximation $\mathrm{T}_{0}(\mathrm{t})$ and several unknown parameters $C_{j}$ and $C_{k} i=1,2, \ldots, n, j=1,2, \ldots, s$, $\mathrm{k}=\mathrm{s}+1, \mathrm{~s}+2, \ldots, \mathrm{n}$ and $\mathrm{P}\left[\mathrm{N}\left(\mathrm{T}_{0}(\mathrm{t})\right]\right.$ is a part of $\mathrm{N}\left[\mathrm{T}_{0}(\mathrm{t})\right]$. The auxiliary function $\mathrm{A}_{1}$ and $\mathrm{A}_{2}$ (namely auxiliary functions) are not unique and are of the same form as $\mathrm{T}_{0}(\mathrm{t})$. If $\mathrm{N}\left[\mathrm{T}_{0}(\mathrm{t})\right]=0$ then it is clear that $\mathrm{T}_{0}(\mathrm{t})$ is an exact solution of Eq.(37).

The unknown parameters $\mathrm{C}_{\mathrm{i}}$ can be optimally identified via different methods, such as Galerkin method, Ritz method, the least square method, colocation method and so on [19-23]. If

$$
R\left(t, C_{i}\right)=L\left[\bar{T}\left(t, C_{i}\right)\right]+N\left[\bar{T}\left(t, C_{i}\right)\right]
$$

where $\bar{T}\left(t, C_{i}\right)$ is given by Eq.(39), then we construct the functional

$$
J\left(C_{1}, C_{2}, \ldots, C_{n}\right)=\int_{(D)} R^{2}\left(t, C_{i}\right) d t
$$

and the values of the parameters $C_{i}$ are obtained from the equations

$$
\frac{\partial J}{\partial C_{1}}=\frac{\partial J}{\partial C_{2}}=\ldots=\frac{\partial J}{\partial C_{n}}=0
$$

Alternatively, an equivalent system of algebraic equations useful for identifying the values of the parameters $\mathrm{C}_{\mathrm{i}}$ would be given by

$$
R\left(t_{1}, C_{i}\right)=R\left(t_{2}, C_{i}\right)=\ldots . R\left(t_{n}, C_{i}\right)=0
$$

By means of this novel method, the first-order approximate solution given by Eq.(39) is well determined after the identification of the optimal values of the initial unknown convergence-control parameters $\mathrm{C}_{\mathrm{i}}, \mathrm{i}=1,2, \ldots, \mathrm{n}$.

It will be proved that our procedure is a powerful tool for solving nonlinear problems without small or large parameters.

\section{Application of OAFM to Eqs. (33) and (36)}

If we introduce the independent variable $\tau=\omega t$ and the dependent variable $\mathrm{T}=\mathrm{Ay}$, then Eqs. (33) and (36) become, respectively

$$
\begin{gathered}
y^{\prime \prime}+\frac{a}{\omega^{2}} y+\frac{b A}{\omega^{2}} y^{2}+\frac{c A^{2}}{\omega^{2}} y^{3}=0 \\
y(0)=1, \quad y^{\prime}(0)=0
\end{gathered}
$$

where prime denotes derivative with respect to $\tau$ and $\omega$ is the frequency of the system.

For Eq.(50) the linear operator may be written

$$
L[y(\tau)]=y^{\prime \prime}+y
$$

and the corresponding nonlinear operator becomes

$$
N[y(\tau)]=\left(\frac{a}{\omega^{2}}-1\right) y+\frac{b A}{\omega^{2}} y^{2}+\frac{c A^{2}}{\omega^{2}} y^{3}
$$

The Eqs.(41) and (42) become

$$
y_{0}^{\prime \prime}+y_{0}=0, \quad y_{0}(0)=1, y^{\prime}(0)=0
$$

and has the solution

$$
y_{0}(\tau)=\cos \tau
$$

Substituting Eqs.(55) into (53), we obtain

$$
\begin{aligned}
& N\left[y_{0}(\tau)\right]=\frac{b A}{2 \omega^{2}}+\left(\frac{a}{\omega^{2}}-1+\frac{3 c A^{2}}{4 \omega^{2}}\right) \cos \tau+ \\
& +\frac{b A}{\omega^{2}} \cos 2 \tau+\frac{c A^{2}}{4 \omega^{2}} \cos 3 \tau
\end{aligned}
$$

The Eq.(46) can be written as

$$
\begin{aligned}
& y_{1}^{\prime \prime}+y_{1}+A_{1}\left(\cos \tau, C_{j}\right) P\left[N\left(y_{0}(\tau)\right)\right]+ \\
& +A_{2}\left(\cos \tau, C_{j}\right)=0, \quad y_{1}(0)=y_{1}^{\prime}(0)=0
\end{aligned}
$$


where

$$
\begin{gathered}
P\left[N\left(y_{0}(\tau)\right)\right]=\frac{b A}{2 \omega^{2}}+ \\
+\left(\frac{a}{\omega^{2}}-1+\frac{3 c A^{2}}{4 \omega^{2}}\right) \cos \tau+\frac{b A}{\omega^{2}} \cos 2 \tau \\
A_{1}\left(\cos \tau, C_{j}\right)=-\left(C_{1}+2 C_{2} \cos \tau+2 C_{3} \cos 2 \tau\right) \\
A_{2}\left(\cos \tau, C_{k}\right)=-\left(C_{4} \cos 3 \tau+C_{4} \cos 4 \tau\right)
\end{gathered}
$$

where $\mathrm{C}_{1}, \mathrm{C}_{2}, \mathrm{C}_{3}$ and $\mathrm{C}_{4}$ are unknown parameters at this moment. However, the function $\mathrm{A}_{1}, \mathrm{~A}_{2}$ and $\mathrm{P}$ are not unique. Alternatively, we may choose these functions in the form:

$$
\begin{gathered}
P\left[N\left(y_{0}(\tau)\right)\right]=\frac{b A}{2 \omega^{2}}+\left(\frac{a}{\omega^{2}}-1+\frac{3 c A^{2}}{4 \omega^{2}}\right) \cos \tau \\
A_{1}\left(\cos \tau, C_{j}\right)=-\left(C_{1}+2 C_{2} \cos \tau\right) \\
A_{2}\left(\cos \tau, C_{k}\right)=-\left(C_{3} \cos 3 \tau+C_{4} \cos 5 \tau\right)
\end{gathered}
$$

or yet

$$
\begin{gathered}
P\left[N\left(y_{0}(\tau)\right)\right]=\frac{b A}{2 \omega^{2}}+\frac{b A}{\omega^{2}} \cos 2 \tau+\frac{c A}{4 \omega^{2}} \cos 3 \tau \\
A_{1}\left(\cos \tau, C_{j}\right)=-\left(C_{1}+2 C_{2} \cos \tau+\right. \\
\left.+2 C_{3} \cos 2 \tau+2 C_{4} \cos 3 \tau\right) \\
A_{2}\left(\cos \tau, C_{k}\right)=0
\end{gathered}
$$

and so on. Using only Eqs. (58), (59) and (60), Eq.(57) becomes

$$
\begin{aligned}
& y_{1}^{\prime \prime}+y_{1}=\frac{b A}{2 \omega^{2}} C_{1}+\left(\frac{4 a+3 c A^{2}}{4 \omega^{2}}-1\right) C_{2}+\frac{b A C_{3}}{2 \omega^{2}}+ \\
& +\left[\frac{3 b A}{2 \omega^{2}} C_{2}+\left(\frac{4 a+3 c A^{2}}{4 \omega^{2}}-1\right)\left(C_{1}+C_{3}\right)\right] \cos \tau+ \\
& +\left[\frac{b A}{\omega^{2}}\left(C_{3}+\frac{1}{2} C_{1}\right)+\left(\frac{4 a+3 c A^{2}}{4 \omega^{2}}-1\right) C_{2}\right] \cos 2 \tau+ \\
& +\left[\left(\frac{4 a+3 c A^{2}}{4 \omega^{2}}-1\right) C_{3}+\frac{b A}{\omega^{2}} C_{2}+C_{4}\right] \cos 3 \tau+ \\
& +\left(\frac{b A}{\omega^{2}} C_{3}+C_{5}\right) \cos 4 \tau \quad y_{1}(0)=y_{1}^{\prime}(0)=0
\end{aligned}
$$

The solution of Eq.(67) is chosen so that to contain no secular terms, which lead to the condition

$$
\omega^{2}=a+\frac{3}{4} c A^{2}+\frac{3}{2} b A \frac{C_{2}}{C_{1}+C_{3}}
$$

The solution of Eq.(67) is given by

$$
\begin{aligned}
& y_{1}\left(\tau, C_{i}\right)=\frac{b A}{2 \omega^{2}} C_{1}+\left(\frac{4 a+3 c A^{2}}{4 \omega^{2}}-1\right) C_{2}+\frac{b A}{2 \omega^{2}} C_{3}+ \\
& +\frac{1}{3}\left[\frac{b A}{2 \omega^{2}}\left(C_{1}+2 C_{3}\right)+\left(\frac{4 a+3 c A^{2}}{4 \omega^{2}}-1\right) C_{2}\right](\cos \tau- \\
& -\cos 2 \tau)+\frac{1}{8}\left[\frac{b A}{2 \omega^{2}} C_{2}+\left(\frac{4 a+3 c A^{2}}{4 \omega^{2}}-1\right) C_{3}+\right. \\
& \left.+C_{4}\right](\cos \tau-\cos 3 \tau)+\frac{1}{15}\left(\frac{b A}{2 \omega^{2}} C_{3}+\right. \\
& +C_{4}(\cos \tau-\cos 4 \tau)
\end{aligned}
$$

From Eqs. (55), (69) and (39) and taking into account that $\tau=\omega t$ and $\mathrm{T}=\mathrm{Ay}$, we can get the first-order approximate solution of Eqs.(33) and (36) in the form

$$
\begin{aligned}
& T(t)=A \cos \omega t+\left[\frac{b A^{2}}{2 \omega^{2}} C_{1}+A\left(\frac{4 a+3 c A^{2}}{4 \omega^{2}}-1\right) C_{2}+\right. \\
& \left.+\frac{b A^{2} C_{3}}{2 \omega^{2}}\right](1-\cos \omega t)+\frac{1}{3}\left[\frac{b A^{2}\left(C_{1}+2 C_{3}\right)}{2 \omega^{2}}+\right. \\
& \left.\left(\frac{4 a A+3 c A^{3}}{4 \omega^{2}}-A\right) C_{2}\right](\cos \omega t-\cos 2 \omega t)+ \\
& +\frac{1}{8}\left[\frac{b A^{2} C_{2}}{2 \omega^{2}}+\left(\frac{4 a A+3 c A^{2}}{4 \omega^{2}}-A\right) C_{3}+A C_{4}\right](\cos \omega t- \\
& -\cos 3 \omega t)+\frac{1}{15}\left(\frac{b A^{2} C_{3}}{2 \omega^{2}}+A C_{5}\right)(\cos \omega t-\cos 4 \omega t)
\end{aligned}
$$

where $\omega$ is given by Eq.(68).

\section{Numerical examples}

We consider the following three cases, in which the parameters a, b and c are obtained from Eqs. (34) and (35):

$$
\begin{aligned}
& a=\pi^{4}+k_{1}+\pi^{2} k_{3}, \quad b=-2 \sqrt{2} \pi^{3} \alpha \lambda \\
& c=\frac{3 k_{2}}{2}+\alpha \pi^{4}
\end{aligned}
$$

\subsection{Case 1}

For $\mathrm{k}_{1}=\mathrm{k}_{3}=50$ and $\mathrm{k}_{3}=25, \alpha=1, \lambda=0.1, \mathrm{~A}=0.6$, the parameters $C_{i}$ and $\omega$ are obtained using the described procedure as

$$
\begin{aligned}
& C_{1}=-0.803588048507, C_{2}=-0.286807831152, \\
& C_{3}=0.165036386496, C_{4}=-0.024155608542, \quad(72) \\
& C_{5}=0.000054512958, \omega=25.9571
\end{aligned}
$$

The first-order approximate solution of Eqs.(33) and 


$$
\begin{aligned}
& \bar{T}(t)=0.000590623+0.598237 \cos \omega t- \\
& -0.0000679857 \cos 2 \omega t+0.00121644 \cos 3 \omega t+ \\
& +0.0000235972 \cos 4 \omega t
\end{aligned}
$$

To emphasize the accuracy of the obtained solution, we compare the approximate result obtained through OAFM with the numerical integration results. Fig.2 presents a comparison of the present solution (72) and numerical results obtained by a fourth-order RungeKutta method.

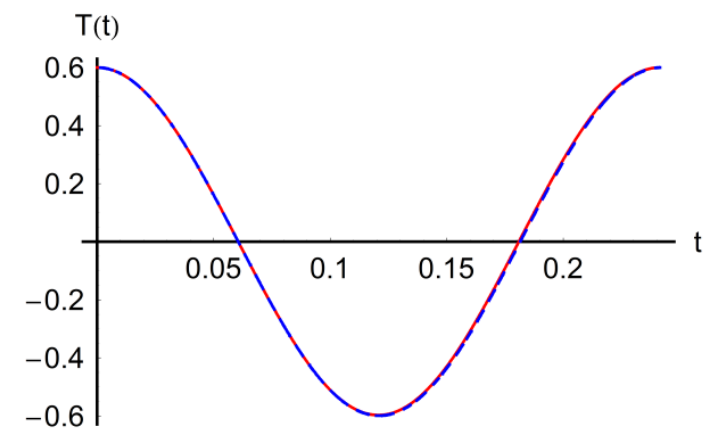

Fig.2. Comparison between the approximate solution (73) and numerical integration results numerical; analytical

\subsection{Case 2} have

In the second case, for $\mathrm{k}_{1}=50, \mathrm{k}_{2}=40$ and $\mathrm{k}_{3}=20$, we

$$
\begin{aligned}
& C_{1}=-0.8086487353162, C_{2}=-0.2991968209976, \\
& C_{3}=0.1707921303513, C_{4}=-0.0271212510421, \quad(74) \\
& C_{5}=0.00005828000552259946, \omega=23.9352
\end{aligned}
$$

and therefore

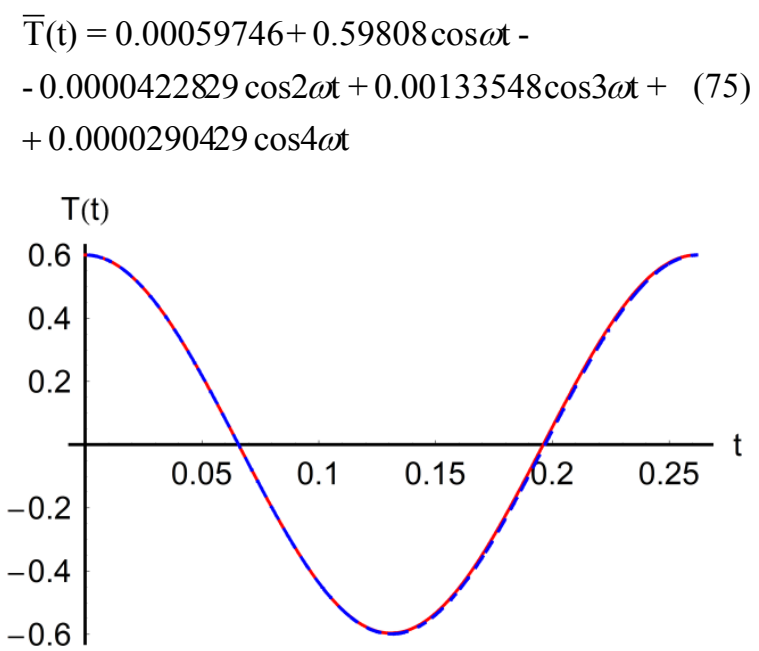

Fig.3. Comparison between the approximate solution (75) and numerical integration results numerical; _ _ _ analytical

\subsection{Case 3}

In the last considered case, $\mathrm{k}_{1}=40, \mathrm{k}_{2}=30$ and $\mathrm{k}_{3}=50$, such that

$$
\begin{aligned}
& C_{1}=-0.8652502750014, C_{2}=-0.34584181383991, \\
& C_{3}=0.18086458531247, C_{4}=-0.0266366276983, \quad(76) \\
& C_{5}=0.000032366254671716754, \omega=25.7944
\end{aligned}
$$

and therefore

$$
\begin{aligned}
& \vec{T}(t)=0.000379825+0.598285 \cos \omega t+ \\
& +0.0000164287 \cos 2 \omega t+0.00129126 \cos 3 \omega t+ \\
& +0.0000273128 \cos 4 \omega t
\end{aligned}
$$

In figs. 3 and 4 are compared the solutions (75) and (77) with the corresponding numerical integration results.

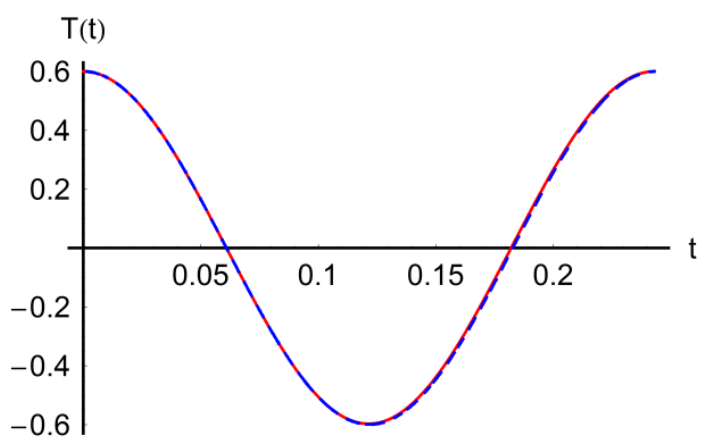

Fig.4. Comparison between the approximate solution (77) and numerical integration results numerical; analytical

From Figs.2-4 one can be observed that the firstorder approximate analytical results obtained by means of OAFM are almost identical with the numerical simulation results in all considered cases for various values of the parameters $\mathrm{k}_{\mathrm{i}}, \mathrm{i}=1,2,3$.

\section{Conclusions}

In this work, we proposed a reliable new technique, namely the Optimal Auxiliary Functions Method (OAFM), which accelerates the rapid convergence of the approximate analytical solution of nonlinear thermomechanical vibration of a FGB on WinklerPasternak foundation.

The proposed procedure is valid even if the nonlinear equation does not contain any small or large parameters. Our construction of the first iteration is different from other traditional approaches especially concerning the optimal auxiliary functions which depend on some initially unknown parameters $\mathrm{C}_{\mathrm{i}}$.

The initially unknown parameters, called convergence-control parameters, after identifying their optimal values, provide a fast convergence of the approximate analytical solutions using only one iteration.

The main advantage of the proposed procedure is the possibility to optimally control and adjust the convergence of the solutions through the auxiliary functions. 
The obtained approximate analytical solutions are in excellent agreement with the numerical integration results. This proves the validity of our approach, and on the other hand, proves that this method is very efficient in practice.

\section{References}

1. V. Birman, L.W. Byrd, AIAA Journal, 45, 2747 (2007)

2. M.C Dokmeci, J. of the Acoustical Society of America, 119, 3336 (2006)

3. M.K. Khorrambadi, J. of Solid Mech. 1, 22 (2009)

4. O. Carl, P. Villamil, C. Zhang, Proc. Appl. Math. Mech., 11, 199 (2011)

5. N.T. Alshabatat, K. Naghshineh, Advances in Acoustics and Vibration, ID 752361, 2014

6. L-L. Ke, J. Yong, S. Kitipornchai, Meccanica 45, 743 (2010)

7. H. Yaghoobi, M. Torabi, J. Theor. Appl. Mech., 51, 39 (2013)

8. N. Mohammadi, Int. J. of Eng. and Tech. Sci., 3, 144 (2015)

9. $\mathrm{Y} \mathrm{Fu}, \mathrm{J}$. Wang, $\mathrm{Y}$ Mao, J. of Intelligent Material Syst. and Struct., 22, 2093 (2011)

10. B. Thomas, P. Inamdar, T. Roy, B.K. Nanda, Int. J. on Theor. and Appl. Res. in Mech. Eng., 2, 97 (2013)

11. M.N. Elmaguri, M. Haterbouch, A. Bouayad, O. Oussouaddi, J. Mater. Environ. Sci., 6, 3620 (2015)

12. J. Yang, S. Kitipornchai, C. Feng, Int. J. of Structural Stability and Dynamics, 15, 1540015 (2015)

13. N.V.R. Maganti, M.R. Nalluri, Int. J. Mech. and Material Eng., 10, 21 (2015)

14. K.R. Kashizadeh, A.A. Asfarjani, Advances in Materials, 5, 57 (2016)

15. G. Yigit, A. Sahim, M. Bayram, Open Math., 14, 661 (2016)

16. Z. Su, G. Jin, T Ye, Smart Materials and Struct., 25, 065003 (2016)

17. L Mu. G. Zhao, Shock and Vibration, ID 3287645 , 2016

18. B.S. Shvartsman, J. Majak, Adv. in Theoretical and Appl. Mech., 9, 31 (2016)

19. S. Emam, A.H. Nayfeh, Composite Structures, 88, 636 (2009)

20. N. Herisanu, V. Marinca, Springer Proc. Math. and Statistics, 182, 169 (2016)

21. V. Marinca, N. Herisanu, Nonlinear dynamical systems in engineering. Some approximate approaches (Springer, Heidelberg, 2011)
22. V. Marinca, N. Herisanu, The optimal homotopy asymptotic method. Engineering applications, (Springer, Cham, 2015)

23. V. Marinca, N. Herisanu, Int. J. Heat and Mass Transf 79, 146 (2014) 